\title{
Implementing the Mean Spherical Approximation model in the speciation code CHEAQS Next at high salt concentrations
}

\author{
Wilko Verweij ${ }^{*}$. Jean-Pierre Simonin ${ }^{2}$ \\ ${ }^{1}$ Deltares, P.O. Box 177, 2600 MH Delft, The Netherlands (wilko.verweij@deltares.nl); ORCID 0000- \\ 0003-0665-4841 \\ ${ }^{2}$ CNRS, Laboratoire PHENIX, Campus P.M. Curie, Sorbonne Université, F-75005, Paris, France
}

Keywords Speciation program $\cdot$ MSA model $\cdot$ activity correction $\cdot$ CHEAQS

\begin{abstract}
Speciation programs all struggle with the challenge of converting equilibrium constants to non-zero ionic strength. Equations like the Davies equation are semi-empirical and do not give satisfactory results at high salt concentrations. The Mean Spherical Approximation (MSA) model is a method with a solid theoretical basis that gives good results at high salt concentrations. We implemented the MSA model in a new 64-bit version of the speciation program CHEAQS Next which is under development. It was shown that the results obtained with this research version are identical to results obtained earlier by Simonin [1]. It is the first time the MSA model is implemented in a production program.
\end{abstract}

\section{Introduction}

Chemical speciation is an important factor in many aqueous processes in the environment. For example, the speciation of an element determines its physico-chemical behaviour by processes like complexation in the solution phase, both by inorganic ligands and by the mixture of naturally occurring organic molecules. Also adsorption onto mineral phases and precipitation are related to the speciation of an element. Redox conditions can have a profound effect on the speciation of elements e.g. in the case of highly soluble iron(II) versus poorly soluble iron(III).

The bioavailability and hence the toxicity of elements for aquatic organisms is also related to their speciation [2]. Especially for metals a lot of effort has been made to understand why an element is toxic in a certain situation but not toxic in another one.

Different experimental approaches have been used to assess the speciation of elements. Physicochemical methods are very useful in unravelling the speciation, but they sometimes lack the required analytic sensitivity. Biological methods can be used to more or less directly assess the effects of an element on organisms, so bypassing the need to quantify the speciation in chemical terms. Modelling is a way to calculate the chemical speciation based on information about total concentrations, equilibrium constants, etc.

Many programs have been developed over time like GEOCHEM [3], MINEQL [4], TiTrator [5], (Visual) MINTEQ [6], ORCHESTRA [7], ECOSAT [8], CHESS [9], JESS [10], and CHEAQS, the latter being developed by one of us (WV). These models calculate the speciation of elements, based on input total concentrations and equilibrium constants. Equilibrium constants are valid at a certain temperature and ionic strength; usually speciation models contain the values of $T=298 \mathrm{~K}$ and a hypothetical zero ionic strength. For conversion from one temperature to another, usually the Van ' $\mathrm{t}$ Hoff equation is used, which requires knowledge of the enthalpy $\Delta \mathrm{H}$ of an equilibrium reaction [11]. For conversion from one value of the ionic strength to another, many different approaches are in use. This conversion is quite important, because already at relatively low ionic strengths, deviations from ideality become significant, and the activity of a species starts to deviate from its concentration. Hence, the intrinsic, or thermodynamic, equilibrium constants (i.e. constants at a hypothetical ionic strength of zero) are no longer valid and the activity $a$ of ions at non-zero ionic strength needs to be calculated from the concentration and the activity coefficient: 
where $\gamma$ is the activity coefficient on concentration scale (dimensionless) and $c$ is the concentration; $a$ and $c$ are in $\mathrm{mol} \cdot \mathrm{L}^{-1}$.

The first solution to calculate activity coefficients for non-zero ionic strength was proposed by Debye and Hückel $[12,13]$. The Debye-Hückel (DH) theory assumes ions are point charges in the cloud around a central ion, so it does not account properly for the volume occupied by these ions, nor for the volume of the shell of ligands and/or water molecules around them. The DH equation gives good approximations up to ca. $0.01 \mathrm{~mol} \cdot \mathrm{L}^{-1}$ for a 1:1-electrolyte, and only up to ca. $0.001 \mathrm{~mol} \cdot \mathrm{L}^{-1}$ for a $2: 2$ electrolyte [14]. When the ionic strength exceeds these values, the values of the activity coefficients predicted by the $\mathrm{DH}$ equation deviate significantly from the experimental ones. Many environmental applications require values for higher ionic strengths making the $\mathrm{DH}$ equation insufficient. To overcome this problem, many researchers have suggested other methods, like the extended DH equation, the Güntelberg equation and the Davies equation [15]. The current (32-bit) version of CHEAQS Next uses the Davies equation, which calculates activity coefficients $\gamma$ as follows:

$\log _{10} \gamma=-0.5079 z^{2}\left(\frac{\sqrt{\mathrm{I}}}{1+\sqrt{\mathrm{I}}}-0.3 \mathrm{I}\right)$ where $z$ is the charge of the species and $I$ is the ionic strength. Sometimes 0.2 instead of 0.3 is used.

It is also worth quoting the Bromley [16] and Pitzer [17] equations, as well as the Specific lon Interaction Theory (SIT) [18], that were all inspired by the Guggenheim equation [13]. The latter consisted of the DH equation plus a correction term linear in the ionic strength. The Bromley [16] and Pitzer [17] equations, and the SIT, were built by adding more correction terms to the DH term which is valid only at low (or very low) ionic strength as mentioned in the previous paragraph. A weak point of these equations is that they are based on an empirical virial-type expansion of the activity coefficient at higher salt concentrations (beyond the DH term). It may be noted that this type of expansion not only accounts for electrostatic effects, but also for non-electrostatic effects such as those arising from excluded volume between ions (in the so-called 'primitive' model) that are clearly not considered in the $\mathrm{DH}$ equation. As a consequence of these features, these equations require the determination of many independent adjustable lumped parameters, as is the case of the Pitzer equation.

Although many of these methods are able to predict the activity of ions fairly well, these empirical extensions lack a firm theoretical basis. Against this backdrop, it must be recognized that, subsequently, other theories have been developed that were based on statistical mechanics, and more precisely integral equation theories. For electrolytes, these theories were the scaled particle theory (SPT) which expresses the contribution of excluded volume to non-electrostatic deviations from ideality [19], and the Mean Spherical Approximation (MSA) [19, 20] for a better account (as compared to DH theory) of the effect of excluded volume on the electrostatic contribution. The combination of SPT and MSA is sufficient for a description of non-idealities in ionic solutions [21-24]; it does not require the use of an additional virial-type expansion.

For the new (64-bit) version of CHEAQS Next that one of us (WV) was preparing, we wanted to reconsider the current choice of the Davies equation. Despite the quality of some of the available equations, we preferred to implement a method with a more solid theoretical basis. The Mean Spherical Approximation model (MSA) is such a method: it is similar to other methods in the sense that it can predict activity coefficients as a function of salt concentration, but different because it does have a solid theoretical basis $[19,20]$. It is also different because the effect of ion charges on the ionic activity coefficients is not expressed in terms of the sole ionic strength. The model takes into account the volume taken up by the ions as well as by the shell of water molecules bound to the ions [22], the main assumption being that the hydrated ions are spheres. Especially when a complex is not 
symmetrical, it is likely that the complex is not strictly spherical. The model is able to predict activity coefficients of ions up to an ionic strength of $3 \mathrm{~mol} \cdot \mathrm{L}^{-1}$ fairly well with constant ion sizes and using the dielectric constant of water [1]. Therefore, we decided to test the MSA model for predicting activity coefficients in multi-component electrolytes, in a 'research version' and then subsequently implement all features of the 32-bit version into this 64-bit version.

The MSA model is quite popular in the community of workers dealing with electrolytes, because it goes beyond the simple DH theory and it is expressed in terms of explicit equations that can be solved analytically for the most part. The MSA has been applied already to a large variety of systems. Some examples are as follows. It has been used for the prediction of activity coefficients of amino acids in aqueous solutions $[25,26]$. Researchers have described the effects of ionic strength on the ionic product of water using the MSA model $[27,28]$. It was employed to predict ion-specific excess chemical potentials in the transfer of $\mathrm{Ca}^{2+}$ ions through cell membrane channels, a very important biological and physiological process [29]. The MSA has been used to get an interpretation of the impact of sugars on ion properties in milk-based systems by taking into account electrostatic interactions and volume exclusion [30]. Lastly, the effect of temperature on the activity coefficients of various salts in water [31], and deviations from ideality in organic electrolyte solutions [32-34] (an industrially relevant topic nowadays) have been obtained in this framework.

Below, we will first describe the MSA model, then give the results of the calculations of the research version of CHEAQS.

\section{Methods}

\subsection{The MSA model}

The MSA model is described in detail by Simonin $[1,21]$. Here we give the formulas to calculate the activity coefficient $\gamma$ for a species $i$.

$\ln \gamma_{i}=\ln \gamma_{i}^{H S}+\ln \gamma_{i}^{e l}$

where $\gamma_{i}$ consists of hard-sphere and electrostatic parts. The hard-sphere part describes effects due to pure volume exclusion, while the electrostatic part takes into account effects of Coulomb interactions between ions.

When the salt concentration is increased, the term $\ln \gamma_{i}^{H S}$ is positive and increases more or less linearly, while the term $\ln \gamma_{i}^{e l}$ is negative, decreases sharply initially, and then more slowly at higher concentration. The sum term $\ln \gamma_{i}$ decreases initially, and then increases (see Fig. 1 for $\left(\mathrm{CdCl}_{2}\right.$ ), as observed experimentally. 


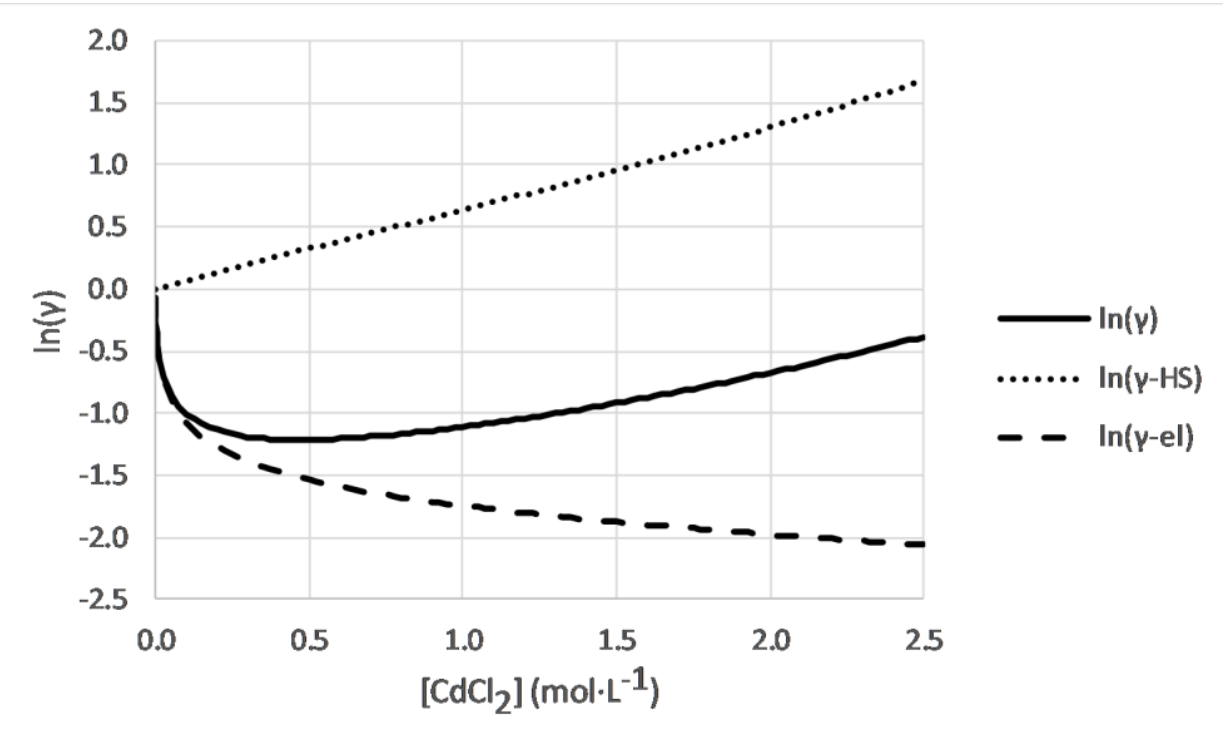

Fig. $1 \mathrm{Ln}(\gamma-\mathrm{HS}), \ln \left(\gamma\right.$-el) and the sum $\ln (\gamma)$ for $\mathrm{Cd}^{2+}$ as a function of $\left[\mathrm{CdCl}_{2}\right]$

The hard-sphere part can be calculated as follows [21]:

$\ln \gamma_{i}^{H S}=-\ln x+\sigma_{i} F_{1}+\sigma_{i}^{2} F_{2}+\sigma_{i}^{3} F_{3}$

where $x$ stands for the free volume fraction in the solution $\left(x=1-X_{3}\right), \sigma$ is the radius of a species (including the hydration shell if any), and $F_{1}, F_{2}$ and $F_{3}$ are defined as follows:

$F_{1}=\frac{3 X_{2}}{x}$

$F_{2}=\frac{3 X_{1}}{x}+3 \frac{X_{2}^{2}}{X_{3} x^{2}}+3 \frac{X_{2}^{2}}{X_{3}^{2}} \ln x$

$F_{3}=\left(X_{0}-\frac{X_{2}^{3}}{X_{3}^{2}}\right) \frac{1}{x}+\frac{3 X_{1} X_{2}-\left(\frac{X_{2}^{3}}{X_{3}^{2}}\right)}{x^{2}}+2 \frac{X_{2}^{3}}{X_{3} x^{3}}-2 \frac{X_{2}^{3}}{X_{3}^{3}} \ln x$

and

$X_{n}=\frac{\pi}{6} \sum_{i} \rho_{i} \sigma_{i}^{n}$

where $\rho$ is the number density of a species, i.e. the number of molecules or ions per $\mathrm{m}^{3}$, and the summation takes place over all solute species in the solution.

The electrostatic part of Eq. 3 can be calculated as follows [21]:

$\ln \gamma_{i}^{e l}=-\frac{\beta e^{2}}{4 \pi \varepsilon \varepsilon_{0}}\left[\frac{\Gamma z_{i}^{2}}{1+\Gamma \sigma_{i}}+\eta \sigma_{i}\left(\frac{2 z_{i}-\eta \sigma_{i}^{2}}{1+\Gamma \sigma_{i}}+\frac{\eta \sigma_{i}^{2}}{3}\right)\right]$

where $\beta=1 /\left(\mathrm{k}_{\mathrm{B}} \mathrm{T}\right), \mathrm{k}_{\mathrm{B}}$ is the Boltzmann constant, $T$ is the temperature (in $\mathrm{K}$ ), $e$ is the elementary charge, $\varepsilon_{0}$ is the permittivity of a vacuum, $\varepsilon$ is the relative permittivity of solution $\left(78.38\right.$ at $\left.25^{\circ} \mathrm{C}\right)$. $\Gamma$ can be calculated as follows: 
$4 \Gamma^{2}=\frac{\beta e^{2}}{\varepsilon \varepsilon_{0}} \sum_{i} \rho_{i}\left(\frac{z_{i}-\eta \sigma_{i}^{2}}{1+\Gamma \sigma_{i}}\right)^{2}$

where an iterative approach is needed because $\Gamma$ occurs on both sides of the equation.

$\eta$ in Eqs. 9 and 10 can be calculated as follows:

$\eta=\frac{1}{\Omega} \frac{\pi}{2 x} \sum_{i} \frac{\rho_{i} \sigma_{i} z_{i}}{1+\Gamma \sigma_{i}}$

where $\Omega$ is given by:

$\Omega=1+\frac{\pi}{2 x} \sum_{i} \frac{\rho_{i} \sigma_{i}^{3}}{1+\Gamma \sigma_{i}}$

In Eq. $11 \Gamma$ is needed for the calculation of $\eta$, while in Eq. 10 it is the other way around. So here a circular reference exists which can only be solved iteratively.

Basically only one property for each species, namely its radius, needs to be known to allow these calculations.

\subsection{The program}

We wrote this research version for 64-bits Windows operating systems with the 64-bits FreeBASIC compiler ( $\underline{w w w . f r e e b a s i c . n e t}$ ) in a mixture of BASIC and $C$. The database was taken from the existing 32-bit CHEAQS-version with some minor changes due to errors discovered during the conversion. In addition, $\mathrm{NaCl}$ (aq) was removed because there is no experimental evidence for this complex [35]. For implementing the MSA model, the radius of species was added to the database (taken from ref. [1]). The research version reads data from an input file, calculates the speciation and writes the output to a .csv-file.

To calculate the speciation, an iterative procedure is needed to solve the set of non-linear equations that one ends up with during speciation calculations. To that end, a combination of two methods was used. When possible, a modified Newton-Raphson-method was employed, similar to proposed by Morel and Morgan [36]. For those cases where this method does not lead to convergence, a linear convergence method was used, modified in such a way that it prevents 'oscillating behaviour', especially in the case of cations at high concentrations having stoichiometric coefficients (much) higher than 1.

\section{Results and discussion}

\subsection{Results}

To test the research version, we created an input set to confirm that the computation results were identical to the previous 32-bit CHEAQS-version. Thus we can assume that the results are computationally correct. To implement the MSA model, the radius for cations, anions and complexes as given by Simonin [1] were added to the database, plus the radius for $\mathrm{Na}^{+}\left(2.897 \times 10^{-10} \mathrm{~m}\right.$ as a result of fitting the activity coefficient of $\mathrm{NaCl}$ up to $3 \mathrm{~mol} \cdot \mathrm{kg}^{-1}$; AARD $=2.5 \%$ ) and Eqs. $3-12$ were incorporated in CHEAQS. For each of the $\mathrm{Zn}$ and $\mathrm{Cd}$ halides $\left(\mathrm{Cl}^{-}, \mathrm{Br}^{-}\right.$and $\left.\mathrm{I}^{-}\right)$studied in ref. [1], we performed a series of 100 calculations with increasing salt concentrations. The $\mathrm{pH}$ was calculated by the program, the calculations according to Eqs. 3 - 12 were carried out for the $\mathrm{Zn}$ - resp. Cd-halide. $\mathrm{H}^{+}$ and $\mathrm{OH}^{-}$were not included in the summations because they are present in negligible concentrations compared to the $\mathrm{Zn}$ and $\mathrm{Cd}$ salts. For the water equilibrium $\left(\mathrm{H}^{+}+\mathrm{OH}^{-} \rightleftharpoons \mathrm{H}_{2} \mathrm{O}\right)$, the Davies equation was used. The output was extended with specific information regarding the MSA-calculations. Since no 
public model is available which includes the MSA model, the results obtained this way were compared with those of ref. [1] and found to be identical (see Fig. 2). This demonstrates that we successfully implemented the MSA model in this research-version of CHEAQS, which will eventually be extended to a full-scale production version.

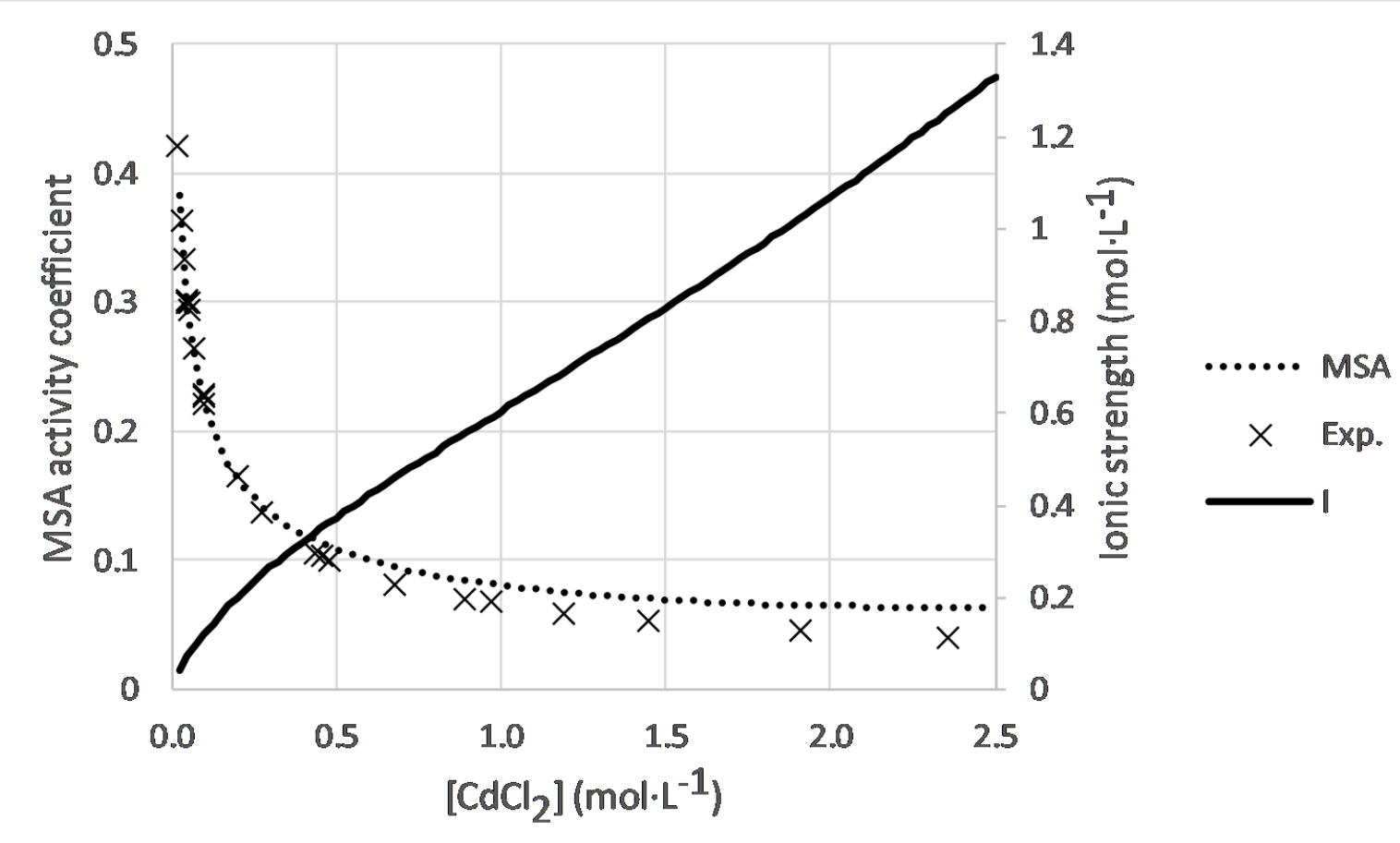

Fig. 2 MSA activity coefficient for $\mathrm{CdCl}_{2}$ (dotted line, left axis) calculated with $\mathrm{CHEAQS}$, experimental data (left axis, from Simonin [1]) and ionic strength I (solid line, right axis) as a function of $\left[\mathrm{CdCl}_{2}\right]$. Results identical to Simonin [1]

To test the environmental relevance, we performed a series of calculations where we added $\mathrm{NaCl}$ to $10^{-7} \mathrm{~mol} \cdot \mathrm{L}^{-1} \mathrm{ZnCl}_{2}$, and the same for $10^{-8} \mathrm{~mol} \cdot \mathrm{L}^{-1} \mathrm{CdCl}_{2}$. In the case of $\mathrm{ZnCl}_{2}$ with $1 \mathrm{~mol} \cdot \mathrm{L}^{-1} \mathrm{NaCl}$ added (which is believed to be the maximum of the range where the Davies equation can be applied), [free $\left.\mathrm{Zn}^{2+}\right]$ was 1.9 times higher when the MSA model was used compared to the Davies equation (see Fig. 3). For $\mathrm{CdCl}_{2}$, [free $\left.\mathrm{Cd}^{2+}\right]$ was 3.3 times higher with the MSA model. At $2 \mathrm{~mol} \cdot \mathrm{L}^{-1}$ of $\mathrm{NaCl}$ the differences get even bigger but that is outside of the range of the Davies equation. For approx. $2 \mathrm{~mol} \cdot \mathrm{L}^{-1}$, we calculated the speciation with MINTEQ which uses the SIT-model; free Cd was calculated to be $3.62 \times 10^{-11} \mathrm{~mol} \cdot \mathrm{L}^{-1}$, almost the same value as the MSA model (see triangle with arrow in Fig. $3 \mathrm{~b}$ ).

The MSA model has therefore proven to give significant results also for environmental conditions, in addition to previous results with laboratory conditions.

Supplementary material is available with more background information about the calculations and convergence procedure. 

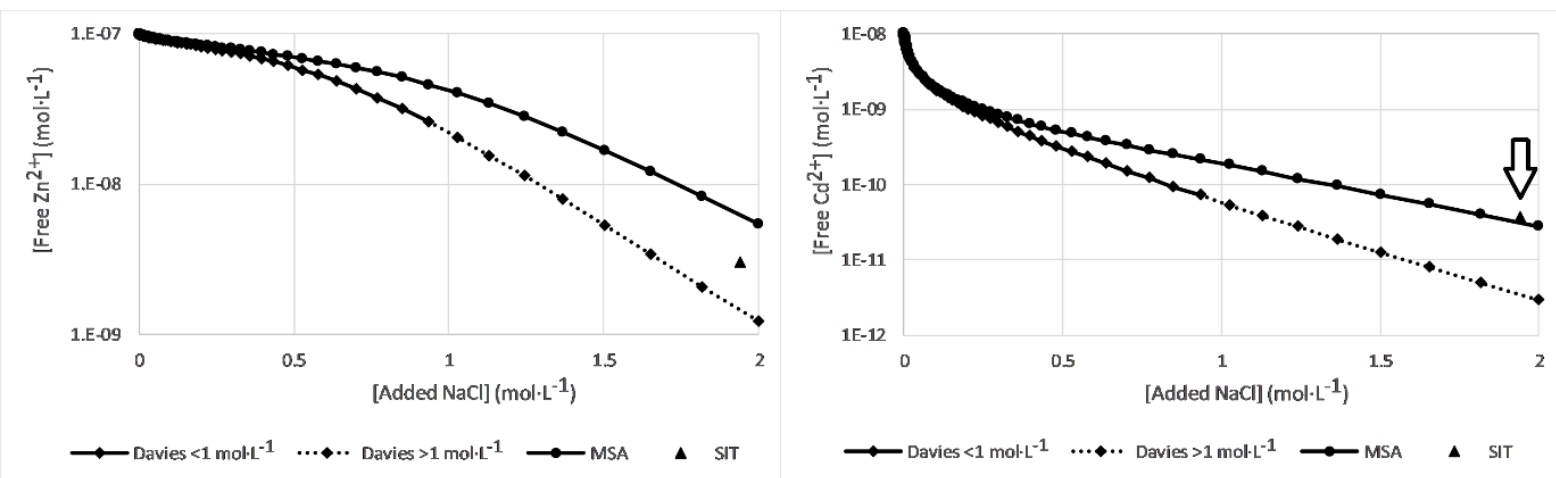

Fig. 3 [Free $\mathrm{Zn}^{2+}$ (left) and [free $\left.\mathrm{Cd}^{2+}\right]$ (right) for increasing [ $\left.\mathrm{NaCl}\right]$ using the Davies equation and MSA model

\subsection{Discussion}

Although now a major step has been set in making the MSA model accessible to a wider group of users, some important further steps still need to be made. First, the accuracy of the present MSA model to describe deviations from ideality in electrolyte mixtures will be examined more closely. A second, more practical, topic is the availability of data. For $\mathrm{Zn}^{2+}, \mathrm{Cd}^{2+}$ and their $\mathrm{Cl}^{-}, \mathrm{Br}^{-}$and I- salts, reliable and consistent values for the effective radii of hydrated ions have been published [1]. Further MSA size parameters for ions will have to be determined and added to the CHEAQS-database. We recommend to focus initially on well-known systems as described in reference books, in order to have sufficient data available for testing the validity of the model. The final task is to add all existing features of the 32-bit version to this 64-bit research version like editing the input interactively, more output features, database manipulation functions and titration capabilities. Updates will be published on the website http://www.cheaqs.eu. The research version is available on the website: http://www.cheaqs.eu/setup64.exe.

For the first time, the MSA model is now included in a public program for calculating chemical equilibria for chemical and environmental samples.

\section{Conclusion}

The MSA model is for the first time incorporated into a production model, so the world of theoretical thermodynamics and practical application of equilibrium modelling have merged: a non-empirical model has been implemented in a speciation program and the MSA model becomes available for a wide audience. As mentioned in the discussion, some steps still need to be made.

Acknowledgement We would like to thank an anonymous reviewer and the editor for their constructive suggestions.

\section{References}

1. Simonin, J.P.: Thermodynamic Consistency In The Modeling Of Speciation In Self Complexing Electrolytes. Ind. Eng. Chem. Res. 56, 9721-9733 (2017)

2. Brezonik, P.L., Arnold, W.A.: Water Chemistry. Oxford University Press Inc. (2011)

3. Shaff, J.E., Schultz, B.A., Craft, E.J., Clark, R.T., Kochian, L.V.: GEOCHEM-EZ: A Chemical Speciation Program With Greater Power And Flexibility. Plant Soil 330, 207-214 (2010)

4. Westall, J.C.: MINEQL: A Computer Program For The Calculation Of Chemical Equilibrium Composition Of Aqueous Systems. Massachusetts Institute Of Technology, Water Quality Laboratory, Cambridge, USA (1976)

5. Cabaniss, S.E.: TITRATOR: An Interactive Program For Aquatic Equilibrium Calculations. Environ. Sci. Tech. 21, 209 - 210 (1976)

6. Gustafsson, J.P.: Visual MINTEQ, available at https://vminteq.Iwr.kth.se/ (2013)

7. Meeussen, H.: ORCHESTRA, available at https://www.ecn.nl/orchestra/ (2009) 
8. Keizer, M.G., Van Riemsdijk, W.H.: ECOSAT User Manual. Wageningen Agricultural University, Wageningen (1998)

9. Van der Lee, J., De Windt, L.: CHESS Tutorial And Cookbook. Users Manual Nr. LHM/RD/02/13, Ecole des Mines de Paris, Fontainebleau, France (2002)

10. May, P.M., Rowland, D., Murray, K., May, E.F.: JESS, available at http://jess.murdoch.edu.au/jess about.shtml (2019)

11. Van 't Hoff, J.H. : Etudes De Dynamique Chimique. Frederik Muller \& Co, Amsterdam (1884)

12. Debye, P., Hückel, E.: Zur Theorie der Elektrolyte. I. Gefrierpunktserniedrigung Und Verwandte Erscheinungen. Phys. Z. 24, 185-206 (1923). Translation available at https://archive.org/details/1923-debye-huckel-2020-braus-translation-english/mode/2up

13. Robinson, R.A., Stokes, R.H.: Electrolyte Solutions: Second Revised Edition. Dover Publications (2012)

14. Wright, M.R.: An Introduction To Aqueous Electrolyte Solutions. John Wiley \& Sons Ltd., Chichester, England (2007)

15. Stumm, W., Morgan, J.J.: Aquatic Chemistry. An Introduction Emphasizing Chemical Equilibria In Natural Waters. John Wiley and Sons, New York (1981)

16. Bromley, L.A.: Thermodynamic Properties Of Strong Electrolytes In Aqueous Solutions. AIChE J., 19, 313-320 (1973)

17. Pitzer, K.S.: Ion Interaction Approach: Theory And Data Correlation. In: Pitzer, K.S. (ed.) Activity Coefficients In Electrolyte Solutions, 2nd edn. C.R.C. Press. Boca Raton (1991), Chap. 3

18. Grenthe, I., Plyasunov, A.V., Spahiu, K.: Estimations Of Medium Effects On Thermodynamic Data. In: Grenthe, I., Puigdomenech, I. (eds.) Modelling in Aquatic Chemistry , . Organisation for Economic Co-operation and Development, Paris (France) (1997), Chap. IX

19. Blum, L.: Primitive Electrolytes in the Mean Spherical Approximation. In: Eyring, H., Henderson, D. (eds.) Theoretical Chemistry: Advances and Perspectives; Academic Press Inc, Vol 5, pp 165 (1980) Chap. 1

20. Blum, L.: Mean Spherical Model For Asymmetric Electrolytes. Mol. Phys. 30, 1529-1535 (1975)

21. Simonin, J.P., Blum, L., Turq, P.: Real Ionic Solutions In The Mean Spherical Approximation. 1. Simple Salts In The Primitive Model. J. Phys. Chem. 100, 7704-7709 (1996)

22. Simonin, J.P.: Real lonic Solutions In The Mean Spherical Approximation. 2. Pure Strong Electrolytes Up To Very High Concentrations, And Mixtures, In The Primitive Model. J. Phys. Chem. B 101, 4313-4320 (1997)

23. Simonin, J.P., Bernard, O., Blum, L.: Real Ionic Solutions In The Mean Spherical Approximation. 3. Osmotic And Activity Coefficients For Associating Electrolytes In The Primitive Model. J Phys Chem B 102, 4411-4417 (1998)

24. Simonin, J.P., Bernard, O., Blum, L.: Ionic Solutions In The Binding Mean Spherical Approximation: Thermodynamic Properties Of Mixtures Of Associating Electrolytes. J. Phys. Chem. B 103, 699704 (1999)

25. Khoshkbarchi, M.K., Vera, J.H.: A Perturbed Hard-Sphere Model With Mean Spherical Approximation For The Activity Coefficients Of Amino Acids In Aqueous Electrolyte Solutions. Ind. Eng. Chem. Res. 35, 4755-66 (1996)

26. Vilariño, T., Sastre de Vicente, M.E.: The Mean Spherical Approximation Methodology Applied To The Acid-Base Equilibria Of Glycine In Artificial Seawater. Phys. Chem. Chem. Phys. 1, 24532456 ( 1999)

27. Sastre de Vicente, M.S., Vilariño, T.: Acid-Base Equilibria in Saline Media: Application of the Mean Spherical Approximation. In: Chemistry of Marine Water and Sediments, Springer, Berlin, Heidelberg (2002), Chap. 11

28. Vilariño, T., Sastre de Vicente, M.E.: Theoretical Calculations Of The lonic Strength Dependence Of The ionic Product Of Water Based On A Mean Spherical Approximation. J. Solution Chem. 26, 833-846 (1997) 
29. Nonner, W., Catacuzzeno, L., Eisenberg. B.: Binding And Selectivity In L-type Calcium Channels: A Mean Spherical Approximation. Biophys. J. 79, 1976-1992 (2000)

30. Gao, R., van Leeuwen, H.P., Temminghoff, E.J., van Valenberg, H.J., Eisner, M.D., van Boekel, M.A.: Effect Of Disaccharides On Ion Properties In Milk-based Systems. J. Agric. Food Chem. 58, 6449-6457 (2010)

31. López-Pérez, G., González-Arjona, D., Molero, M.: Estimation Of Activity Coefficients At Different Temperatures By Using The Mean Spherical Approximation. J. Electroanal. Chem. 480, 9-17 (2000)

32. Fawcett, W.R., Tikanen, A.C.: Application Of The Mean Spherical Approximation To The Estimation Of Electrolyte Activity Coefficients In Methanol Solutions. J. Mol. Liq. 73, 373-384 (1997)

33. Simonin, J.P., Bernard, O.: Organic Electrolyte Solutions: Modeling Of Deviations From Ideality Within The Binding Mean Spherical Approximation. Fluid Phase Equilib. 468, 58-69 (2018)

34. Crothers, A.R., Radke, C.J., Prausnitz, J.M.: 110th Anniversary: Theory of Activity Coefficients for Lithium Salts in Aqueous and Nonaqueous Solvents and in Solvent Mixtures. Ind. Eng. Chem. Res. 58, 18367-18377 (2019)

35. Fuoss, R.M.: Nomenclature For Conductance. J. Chem. Educ. 32, 527 (1955)

36. Morel, F.M.M., Morgan, J.: A Numerical Method For Computing Equilibria In Aqueous Chemical Systems. Environ. Sci. Tech., 6, 58-67 (1972) 